\title{
Long-memory processes in ozone and temperature variations at the region $60^{\circ} \mathrm{S}-60^{\circ} \mathrm{N}$
}

\author{
C. Varotsos ${ }^{1,2}$ and D. Kirk-Davidoff ${ }^{2}$ \\ ${ }^{1}$ Department of Applied Physics, University of Athens, Athens, Greece \\ ${ }^{2}$ Department of Atmospheric and Oceanic Science, University of Maryland, USA
}

Received: 21 February 2006 - Published in Atmos. Chem. Phys. Discuss.: 30 May 2006

Revised: 1 September 2006 - Accepted: 1 September 2006 - Published: 12 September 2006

\begin{abstract}
Global column ozone and tropospheric temperature observations made by ground-based (1964-2004) and satellite-borne (1978-2004) instrumentation are analyzed. Ozone and temperature fluctuations in small time-intervals are found to be positively correlated to those in larger timeintervals in a power-law fashion. For temperature, the exponent of this dependence is larger in the mid-latitudes than in the tropics at long time scales, while for ozone, the exponent is larger in tropics than in the mid-latitudes. In general, greater persistence could be a result of either stronger positive feedbacks or larger inertia. Therefore, the increased slope of the power distribution of temperature in mid-latitudes at long time scales compared to the slope in the tropics could be connected to the poleward increase in climate sensitivity predicted by the global climate models. The detrended fluctuation analysis of model and observed time series provides a helpful tool for visualizing errors in the treatment of long-range correlations, whose correct modeling would greatly enhance confidence in long-term climate and atmospheric chemistry modeling.
\end{abstract}

\section{Introduction}

It has become clear that prediction of global climate change, and of change in the atmosphere's ozone distribution, is impossible without consideration of the complexity of all interactive processes including chemistry and dynamics of the atmosphere (Jacovides et al., 1994; Dameris et al., 1998, 2005, 2006; Sander, 1999; Crutzen et al., 1999; Lawrence et al., 1999; Kirk-Davidoff et al., 1999; Kondratyev and Varotsos,2000, 2001a,b; Ebel, 2001; Varotsos et al., 2001; Brandt et al., 2003; Stohl et al., 2004; Grytsai et al., 2005, Schulz et al., 2001).

Correspondence to: C. Varotsos

(covar@phys.uoa.gr)
In an attempt to shed light on aforementioned complexity, scaling analysis of atmospheric data certainly is becoming an important approach (Lovejoy, 1982; Koscielny-Bunde et al., 1998; Syroka and Toumi, 2001; Tuck et al., 2002, 2003, 2004; Tuck, 2006). Schertzer and Lovejoy (1985) originated the application of scaling to the atmosphere, although its power law roots can be traced back to Richardson (1926).

Let it be recalled that Tuck and Hovde (1999) by applying a measure from fractal geometry to time series of airborne observations of ozone and meteorological quantities in the lower stratosphere suggested that ozone and horizontal wind speed behave as random, self-affine fractals (having anisotropic scaling, in distinction from isotropic scaling in self-similarity). In addition, they found that horizontal wind direction behaves persistently (positive correlation among neighboring time intervals), being thus a candidate for multifractality. To reach these conclusions, Tuck and Hovde (1999), calculated the Hurst exponent (Hurst, 1951), which is relatable to fractal measures by employing the rescaled range analysis (R/S) (Tuck et al., 2003).

Consideration of the decay of correlations in time has often yielded insight into the dynamics of complex systems. A randomly forced first-order linear system with memory should have fluctuations whose autocorrelation decays exponentially with lag time, but a higher order system will tend to have a different decay pattern. However, direct calculation of the autocorrelation function is usually not the best way to distinguish among different decay patterns at long lags, due to noise superimposed on the data (Stanley, 1999; Kantelhardt et al., 2002; Galmarini et al., 2004). The pattern of the decay of autocorrelation with increasing time lag can be obtained using detrended fluctuation analysis (DFA) introduced by Peng et al. (1994), which transforms this decay with increasing lag into an increase in noise amplitude as time scale increases, a measure that is less sensitive to statistical errors (Talkner and Weber, 2000).

Published by Copernicus GmbH on behalf of the European Geosciences Union. 
The main purpose of the present paper is to examine and compare, using DFA, the long-range correlations of fluctuations of total ozone (TOZ) and tropospheric brightness temperature (TRT) and to determine if they exhibit persistent long-range correlations. This means that TOZ or TRT fluctuations at different times are positively correlated, and the corresponding autocorrelation function decays more slowly than exponential decay with increasing lag, possibly by a power-law decay. In other words, "persistence" refers to the "long memory" within the TOZ (or TRT) time series (Stanley, 1999; Hu et al., 2001; Fraedrich and Blender, 2003; Blender and Fraedrich, 2003; Collette and Ausloos, 2004; Varotsos, 2005). Other scientists (e.g. Talkner and Weber, 2000) have previously investigated the decay of autocorrelation in surface temperature records at individual stations, but not in mid-tropospheric temperatures averaged over large regions. Camp et al. (2003) have carried out an empirical orthogonal function study of the temporal and spatial patterns of the tropical ozone variability and have calculated the quantitative contribution of the low-frequency oscillations (e.g. quasi-biennial oscillation - QBO, El Nino-Southern Oscillation - ENSO, decadal oscillation) to the variance of the ozone deseasonalized data, but do not discuss the decay of autocorrelation. The reason that we have chosen these two data sets to explore the memory effects is because these are closely connected with two crucial environmental problems, notably: the ozone depletion and the global warming.

\section{Method and data analysis}

The steps of DFA, which has proved useful in a large variety of complex systems with self-organizing behaviour (e.g. Peng et al., 1994; Weber and Talkner, 2001; Chen et al., 2002; Varotsos et al., 2003a,b; Maraun et al., 2004; Varotsos, 2005), are as follows:

1) We construct a new time series, by integrating over time the deseasonalized time series (of TOZ or TRT). More precisely, to integrate the data, we find the fluctuations of the $\mathrm{N}$ observations $\mathrm{Z}$ (i) from their mean value $Z_{\text {ave }}$ notably: $Z(i)$ $-Z_{\text {ave }}$. Then we construct a new time series (the integrated time series), which is consisting of the following points:

$y(1)=\left[Z(1)-Z_{\text {ave }}\right]$,

$y(2)=\left[Z(1)-Z_{\text {ave }}\right]+\left[Z(2)-Z_{\text {ave }}\right], \ldots$,

$y(k)=\sum_{i=1}^{k}\left[Z(i)-Z_{\text {ave }}\right]$

The integration exaggerates the non-stationarity of the original data, reduces the noise level, and generates a time series corresponding to the construction of a random walk that has the values of the original time series as increments. The new time series, however, still preserves information about the variability of the original time series (Kantelhardt et al., 2002).
2) In the following, we divide the integrated time series into non-overlapping boxes (segments) of equal length, $n$. In each box, a least squares line is fit to the data, which represents the trend in that box. The $y$ coordinate of the straight line segments is denoted by $y_{n}(k)$.

It is worth noting that the subtraction of the mean is not compulsory, since it would be eliminated by the later detrending in the third step anyway (Kantelhardt et al., 2002). The least squares line in each box may be replaced with a polynomial curve of order $l$, in which case the method is referred to as DFA- $l$.

3) Next we subtract the local trend, in each box and calculate, for a given box size $n$, the root-mean-square fluctuation function:

$F(n)=\sqrt{\frac{1}{N} \sum_{k=1}^{N}\left[y(k)-y_{n}(k)\right]^{2}}$

Within each segment the local trend of the random walk is subtracted from the random walk of that segment.

4) We repeat this procedure for different box sizes (over different time scales) to find out a relationship between $F(n)$ and the box size $n$. It is apparent that $F(n)$ will increase with increasing box size $n$. A linear relationship on a log$\log$ plot with slope $\alpha$ indicates the presence of scaling (selfsimilarity), i.e. the fluctuations in small boxes are related to the fluctuations in larger boxes in a power-law fashion and then the power spectrum function $S(f)$ scales with $1 / f^{\beta}$, with $\beta=2 \alpha-1$. Since for stationary time series the power spectrum is the Fourier transform of the autocorrelation function, one can find the following relationship between the autocorrelation exponent $\gamma$ and the power spectrum exponent $\beta: \gamma=1-\beta=2-2 \alpha$, where $\gamma$ is defined by the autocorrelation function $C(\tau)=1 / \tau^{\gamma}$ ( $\tau$ is a time lag) and should satisfy $0<\gamma<1$ (Talkner and Weber, 2000; Chen et al., 2005).

An exponent (slope of log-log plot) $\alpha \neq 0.5$ in a certain range of $n$ values implies the existence of long-range correlations in that time interval, while $\alpha=0.5$ corresponds to the classical random noise. If $0<\alpha<0.5$, power-law anticorrelations are present (antipersistence). When $0.5<\alpha \leq 1.0$, then persistent long-range power-law correlations prevail (the case $\alpha=1$ corresponds to the so-called $1 / f$ noise). $\alpha>1$ implies that the long-range correlations are stronger than in the previous case with $\alpha=1.5$ corresponding to Brownian motion (e.g. Ausloos and Ivanova, 2001).

A detailed discussion on the relation between the variability measure $F(n)$ and the power spectral density (or equivalently to the autocovariance) is presented in Talkner and Weber (2000). For example, a power spectral density that diverges algebraically as $f$ vanishes, $S(f) \sim 1 / f^{\beta}$, results in a scaling behaviour of $F^{2}(n) \sim n^{1+\beta}$ for large $n$. If the power spectral density algebraically decays at large frequencies $S(f) \sim 1 / f^{\beta}$, the detrended variability measure increases at small values of $n$ according to the power law $F^{2}(n) \sim n^{1+\beta}$ provided that $\beta<3$. A power spectral density that decays 
faster than $S(f) \sim 1 / f^{3}$ yields $F^{2}(n) \sim n^{4}$ for small values of $n$ (Talkner and Weber, 2000).

The change of the slope of the DFA in the small scales can be a result of a change of the power law exponent of the power spectrum as in the models presented by Talkner and Weber (2000). There one can see that the relation between alpha and the power law exponent of the power spectrum is not only asymptotically (see Fig. 3 and Eq. 3.15 of Talkner and Weber, 2000). For a piecewise power-law behavior of the power spectrum, the slope of the DFA conforms to the corresponding power-law in each region. The autocorrelation function on the other hand suffers from oscillations which mask the presence of the intermediate scaling region (see Fig. 1 of Talkner and Weber, 2000). This is the reason why we preferred DFA to other conventional methods. The asymptotic behavior results only in the case where distinct time scales are present, as in the case of Markovian random telegraph signals (Varotsos et al., 2002, 2003a, b) or in the case of the autoregressive processes (AC S2602 Varotsos and Kirk-Davidoff, 2006 ). Even in such a case, however, the DFA slope changes continuously (AC S2602 Varotsos and Kirk-Davidoff, 2006b).

\section{Results and discussion}

\subsection{The time scaling of the total ozone fluctuations}

We begin with the investigation of the time scaling of the TOZ fluctuations over the tropical and mid-latitudinal zones of both hemispheres. Figure 1a shows the monthly mean TOZ values over the belt $25^{\circ} \mathrm{S}-25^{\circ} \mathrm{N}$ as derived from the daily TOZ observations of the WMO Dobson Network (WDN) during 1964-2004. It should be clarified that the zonal averaging was based on the daily ground-based observations (daily ozone values are averaged from $25^{\circ} \mathrm{S}$ to $25^{\circ} \mathrm{N}$ ). Inspection of Fig. 1a shows that this time series is apparently non-stationary, and includes both periodic and aperiodic fluctuations.

We start the analysis of this time series by asking if the TOZ value in a given instant has any correlation with the TOZ in a later time, i.e. if TOZ time series exhibits longrange correlations. This question stems from the observation that many environmental quantities have values that remain residually correlated with one another even after many years (long-range dependence). Interestingly, the correlation function of the TOZ time series (as deduced from the power spectral density shown in Fig. 1b) decays more slowly than the corresponding exponential one. The departure from the exponential fit becomes more pronounced towards the low frequencies. Moreover, the determination of the power spectrum is hampered by large statistical uncertainties if one goes to low frequencies e.g. the QBO (with period $\sim 2-\mathrm{yr}$ ), and the 11-yr solar cycle.

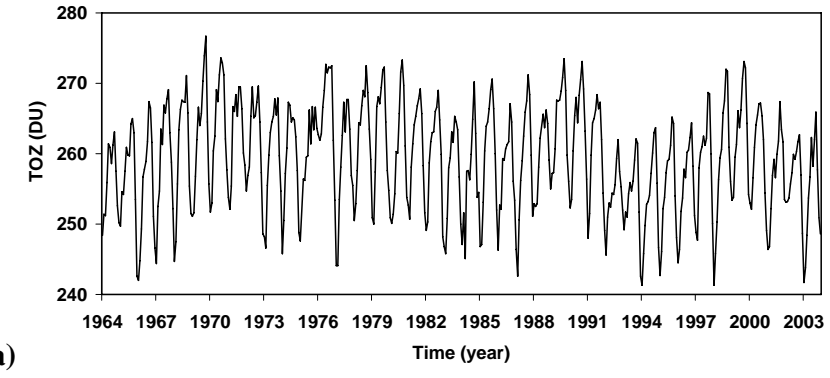

(a)

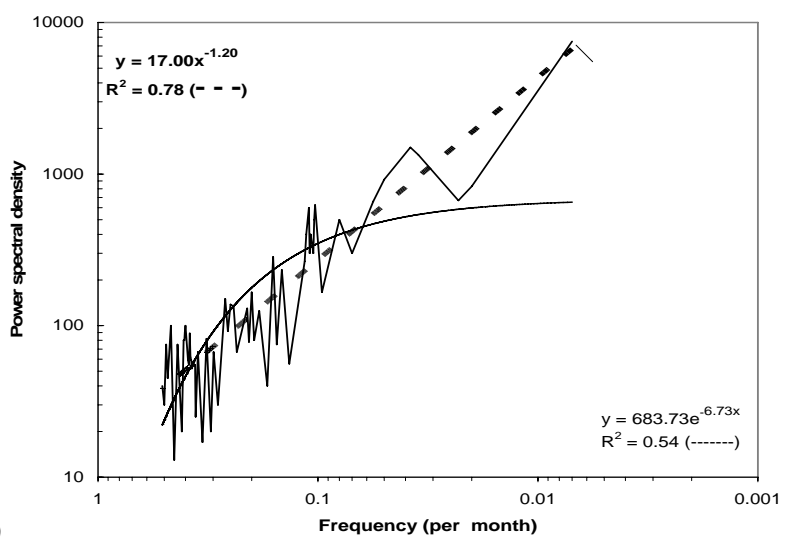

Fig. 1. (a) Total ozone (TOZ) mean monthly values (in Dobson Units - DU) during 1964-2004, over the belt $25^{\circ} \mathrm{S}-25^{\circ} \mathrm{N}$ derived from the WMO Dobson Network $(100 \mathrm{DU}=1 \mathrm{~mm}$ thickness of pure ozone on the Earth's surface). (b) Power spectrum density of TOZ. It is characterized by large statistical uncertainties to low frequencies. Interestingly, from about the 4-month wave to the 11-year solar cycle the power-law fits better $(-1.2 \pm 0.03)$ than the exponential.

To reliably gain insight into this problem, we deseasonalize the data (to avoid obscuring of a possible scaling behaviour from the long-term trend and various frequency peaks induced from the well known cycles - e.g., Steinbrecht et al., 2003; Steinbrecht et al., 2006) (Hu et al., 2001). We then analyze the deseasonalized TOZ (D-TOZ) data of Fig. 1a, using DFA.

In Fig. 2b, a log-log plot of the root-mean-square fluctuation function $F_{d}(\Delta t)=F(n)$ is shown, by applying the first order DFA (DFA-1) to the D-TOZ data derived from WDN, over the belt $25^{\circ} \mathrm{S}-25^{\circ} \mathrm{N}$. Since, $\alpha=1.1( \pm 0.04)$, we conclude that TOZ fluctuations over the tropics exhibit persistent long-range correlations ( $1 / f$ noise-like) for the interval time ranging from about 4 months to 11 years. The long-range correlations obtained do not signify the presence of cycles with definite periodicities (i.e. as described in Camp et al., 2003), but rather the existence of dynamical links between long and short time-scale behavior.

We note that the data treated here are deseasonalized data and much of the influence of QBO and ENSO is removed. These oscillations, when superimposed on the data distort 


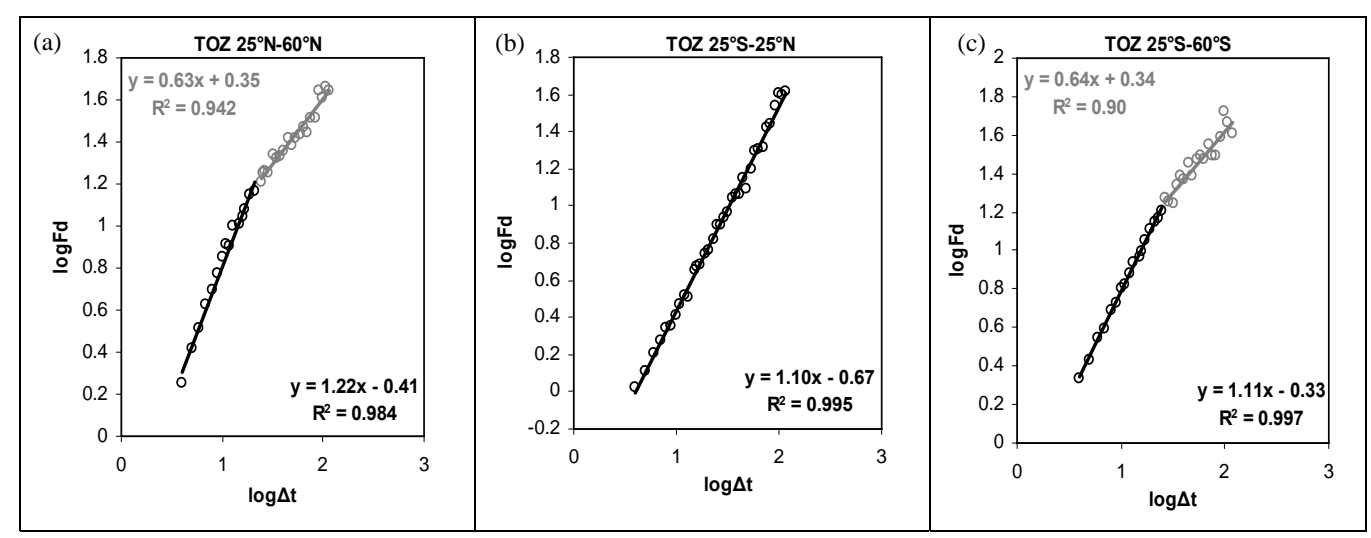

Fig. 2. Log-log plot of the total ozone (TOZ) root-mean-square fluctuation function $(F d)$ versus temporal interval $\Delta t$ (in months) for deseasonalized TOZ values, observed by the WMO Dobson Network over the tropics (b), and mid-latitudes of both hemispheres (a, c) during 1964-2004 (crossover at $\Delta t \approx 28$ months over the mid-latitudes). For comparison reasons see Figs. 2 and 3 of Varotsos (2005).

the linear relation in the log-log plot of the DFA (Hu et al., 2001), but the resulting behavior is opposite to that observed in our data. If there were an influence from periodic oscillations, the slope of the DFA fluctuation measure should increase with increasing time scale rather than decreasing (see Fig. 6 of Hu et al., 2001) below the period of the trend; for time scales just larger than this period, the slope should abruptly become zero. Thus, when comparing Fig. 6 of Hu et al. (2001) with Fig. 2a, for example, we see that the presence of the influence from periodic oscillations should be excluded (AC S2602 Varotsos and Kirk-Davidoff, 2006b).

Next, we examine the extra-tropics and the mid-latitude zone, and specifically the TOZ data of WDN for the latitude belts $25^{\circ} \mathrm{N}-60^{\circ} \mathrm{N}, 25^{\circ} \mathrm{S}-60^{\circ} \mathrm{S}$ during 1964-2004. By employing the DFA-1, the analysis of the D-TOZ data of $25^{\circ} \mathrm{N}-60^{\circ} \mathrm{N}$ shows that, once again, persistent long-range correlations exist. According to Fig. 2a, since $\alpha_{1}=1.22 \pm 0.04$ (for time scales shorter than about 2 years) the correlations in TOZ fluctuations exhibit "stronger memory" compared to that of $\alpha_{2}=0.63 \pm 0.04$ (for time scales from about 2 to 11 years).

The results obtained from the application of the DFA-1 method to the D-TOZ values in the latitude belt $25^{\circ} \mathrm{S}-60^{\circ} \mathrm{S}$ are depicted in Fig. 2c, where again, persistence of TOZ fluctuations is observed. In particular, for time scales shorter than about 2 years, $\alpha_{1}=1.11 \pm 0.02$, while for longer time scales, $\alpha_{2}=0.64 \pm 0.06$. Thus, the tropics exhibit stronger persistence at long time scales than the extratropics, but approximately equivalent persistence at short time scales (Varotsos, 2005).

It is worth noting that, we also applied DFA to the residual TOZ time-series (with QBO, ENSO and 11-year solar cycle intentionally removed) and the results obtained, found to agree fairly well with those presented above.
The fact that in both extratropical bands, the persistence of ozone fluctuations changes character at about 2 years, suggests some connection with the QBO. The result suggests that for timescales longer than 2 years, there is little dynamical memory for ozone fluctuations in the extratropics. It is therefore interesting and puzzling that this change in character of the persistence does not appear in the tropics, where QBO dynamics originate, and where positive TOZ deviations are observed to occur a few months before the maximum westerlies at $50 \mathrm{hPa}$ (WMO, 2003). Similar to the abovementioned results for the TOZ variability over extra-tropics and mid-latitudes are also found by applying the DFA-1 method to the D-TOZ data derived from the Total Ozone Mapping Spectrometer (TOMS) observations (not shown).

To check the above-discussed results, the DFA- $l$ method was applied to the same D-TOZ time series. The results obtained did not show any significant deviations from DFA-1. As a further check, we investigated whether the persistence found in TOZ time series stems from the values of TOZ by themselves and not from their time evolution. Therefore, we applied DFA-1 to randomly shuffled TOZ data over the tropics. This gave $\alpha=0.51 \pm 0.01$. Thus, the persistence in TOZ time series stems from the sequential ordering of the TOZ values and is not a result of the distribution of the TOZ values. Similar results were also obtained for the TOZ time series over extra-tropics and mid-latitudes in both Hemispheres.

In addition, an effort has been made to detect whether the persistence observed in the TOZ data over the tropics and mid-latitudes also characterizes the ozone layer over the polar, Arctic and Antarctic regions (as defined in Hudson et al., 2003). To this end, we constructed a mixed TOZ time series consisting of TOMS observations, when WDN had no available observations for the region of interest, and vice-versa. The application of the same analysis described above led to 
$\alpha=0.83 \pm 0.02$ for the polar region, and to $\alpha=0.81 \pm 0.03$ for the arctic region. Similar results were also obtained for the Southern Hemisphere. Nevertheless, the latter must be considered with caution, because at these regions the number of WDN stations is very limited and TOMS data are only available when sunlight is present.

Furthermore, the log-log plot derived from the application of DFA- 1 on the global D-TOZ data reveals that $\alpha=1.1 \pm 0.02$, suggesting that strong persistence in the tropics and midlatitudes discussed above dominates the variability of the global ozone layer. In summary, the TOZ fluctuations over the tropics, extra-tropics, and mid-latitudes of both hemispheres, as well as globally, exhibit persistent long-range correlations for all time lags between about 4 months- 11 years. Over the extra-tropics, this persistence becomes weaker for time lags between about 2-11 years. These findings are consistent with the preliminary results presented in Varotsos (2005). Comparing, however, the error bars of the DFA slope in Fig. 2 with Figs. 2 and 3 of Varotsos (2005) small differences are observed. It may be attributed to the fact that for the zonal averaging of the TOZ data in Varotsos (2005) the monthly mean ground-based observations were used, instead of the daily ground-based observations that employed in this paper.

Some might argue that Figs. 2 and 4 are evidence for selfsimilar behaviour in ozone and temperature oscillations. We do not make this claim here. Rather we argue that the DFA analysis makes very clear the different behaviour of the tropical and extratropical time series as the time scale increases. It is not essential to our claims or to the interest of the climate community that the behaviour shown in Fig. 2a be appropriately described as self-similar, with two power laws in two different frequency domains. Our point is that at time scales longer than about two years, the behaviour in the tropics and in the extratropics is different and that DFA analysis makes this very clear (Rust, 2006; Varotsos and KirkDavidoff, 2006a, and references therein).

3.2 Time scaling of the tropospheric temperature fluctuations

We next examine the existence of time scaling of the TRT fluctuations, an atmospheric parameter that is often used to quantify global warming. The data used (see for instance Fig. 3) are the passive microwave temperature soundings from the Microwave Sounding Units (MSU channel 2 from the satellites TIROS-N, NOAA-6 to NOAA-12, and NOAA14) and the AdvancedMicrowave Sounding Units (AMSU channel 5 from the satellites NOAA-15to NOAA-17 and AQUA) for the time period 1978-2004.

A detailed description of the multi-satellite data analysis and the development technique of the time series of the biascorrected globally averaged daily mean (pentad averaged) TRT is given in Vinnikov and Grody (2003). It should be recalled that the MSU channel 2 brightness temperature mea-

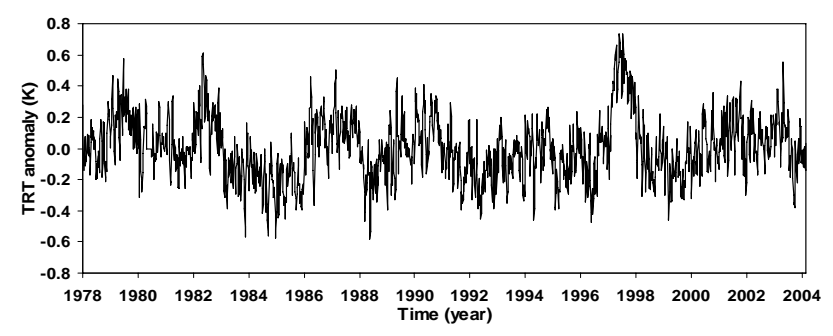

Fig. 3. Time series of the daily mean (pentad averaged) tropospheric brightness temperature (TRT) over the belt $25^{\circ} \mathrm{S}-25^{\circ} \mathrm{N}$, as derived from multi-satellite observations during 1978-2004.

surements have been used during the last fifteen years as an indicator of air temperature in the middle troposphere (Vinnikov and Grody, 2003). Furthermore, to account for the effects of dynamical perturbations on TOZ, the TRT is often used as an index of dynamical variability (Chandra et al., 1996).

In Fig. 4b, a log-log plot of the function $F_{d}(\Delta t)$ is shown, by employing the DFA- 1 to the D-TRT time series averaged in pentads of days over the belt $25^{\circ} \mathrm{S}-25^{\circ} \mathrm{N}$. Since $\alpha_{1}=1.13 \pm 0.04$ (for time scales shorter than about 2 years) the fluctuations in TRT exhibit long range correlations, whilst for time scales from about 2 to 7 years (e.g. El Nino-Southern Oscillation) they obey a random walk $\left(\alpha_{2}=0.50 \pm 0.04\right)$. The crossover point (at about 2 years) was defined as that point where the errors in both linear best fits are minimized.

We now focus on the extra-tropics and the mid-latitude zone, e.g. $25^{\circ} \mathrm{N}-60^{\circ} \mathrm{N}$ and $25^{\circ} \mathrm{S}-60^{\circ} \mathrm{S}$. The application of the DFA-1 to the D-TRT data over both belts (Fig. 4a, c) reveals that long-range power-law correlations exist in both Hemispheres, for the interval time ranging from about 20 days to 7 years, with $\alpha=0.80 \pm 0.01$. Thus the global TRT fluctuations exhibit long-range persistence. These results were confirmed by DFA-2 to DFA-7 analysis yielding $\alpha$-values ranging from 0.78 to 0.86 . We again confirmed the persistence found above by applying DFA-1 on the shuffled TRT anomalies, which again showed no persistent fluctuations.

In our reply (Varotsos and Kirk-Davidoff, 2006b) to the comment by Rust (2006) we have shown that any combination of autoregressive processes violates the statistics of our data. On the use of DFA for the small data lengths investigated here, we refer to the study of Audit et al. (2002), which showed that DFA is the best estimator for the scaling properties.

As concerns the question on why the DFA slope $\alpha$ can exceed unity, we refer to the well known example (e.g. see Varotsos et al., 2006, and references therein) of fractional Gaussian noise (fGn) and fractional Brownian motion (fBm). An $\mathrm{fBm}$ of a given $\mathrm{H}(0<\mathrm{H}<1)$ is non-stationary and exhibits a DFA slope $\alpha=1+\mathrm{H}$, while an fGn (i.e., the time-series of the increments of an $\mathrm{fBm}$ ) is stationary and exhibits a DFA slope $\alpha=\mathrm{H}$ (AC S2602 Varotsos and Kirk-Davidoff, 2006b). 


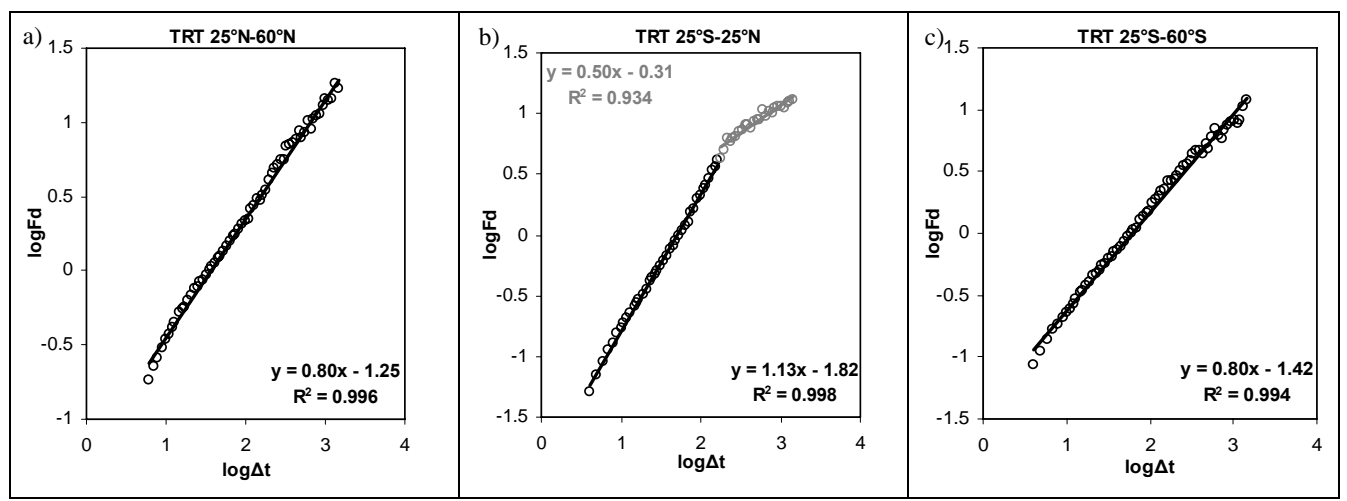

Fig. 4. Log-log plot of the tropospheric brightness temperature (TRT) root-mean-square fluctuation function $(F d)$ versus temporal interval $\Delta t$ (averaged in pentads of days) for deseasonalized TRT (mid-tropospheric temperature) values, observed by the multi-satellite instrumentation over the tropics (b), and mid-latitudes of both hemispheres (a, c) during 1978-2004 (crossover at $\Delta t \approx 28$ months over the tropics).

Future work by the authors will aim to resolve the source of persistence in stratospheric ozone (whether due to forcing from the surface or to internal dynamics) through the inclusion of additional variables in the DFA analysis.

\section{Conclusions}

We find opposite results for mid-tropospheric temperature and for ozone fluctuations. For ozone fluctuations, persistence at long time scales is strongest in tropics, but weaker in mid-latitude, while temperature fluctuations show strong persistence in mid-latitudes, and random noise in the tropics. Various mechanisms might account for the differences we have detected in the persistence in temperature and ozone. Greater persistence is in general a result of either stronger positive feedbacks or larger inertia. Thus, the reduced slope of the power distribution of temperature in the tropics at long time scales, compared to the slope in the mid-latitudes could be connected to the poleward increase in climate sensitivity (due to latitude-dependent climate feedbacks) predicted by the global climate models. However this prediction applies to surface temperature, rather than to mid-tropospheric temperatures, which are expected to increase more or less uniformly with latitude.

We can rationalize the latitude dependence of the persistence in ozone fluctuations as follows. Zonal mean TOZ fluctuations in the mid-latitudes are governed largely by motions of the jet stream, which marks the boundary between low tropopause heights on the poleward side (and more TOZ) and higher tropopause heights (and less TOZ) on the tropical side (Hudson et al., 2003). Such variations might be expected to show relatively low persistence beyond time scales of a few months (or else seasonal weather prediction would be easier). Thus, the difference in persistence patterns between ozone and temperature could arise because the TOZ distribution is more closely tied to gradients in temperature (which are associated with the jet position), than to the temperature itself.

Nowadays, although many coupling mechanisms between ozone and temperature are known, the net effect of the interactions and feedbacks is only poorly understood and quantified. DFA analysis offers a visually clear way to display the long-range correlations of data sets. We have shown that temperature and total ozone averaged over large regions have substantially different patterns of long-range correlations over a range of time scales. Models that hope to predict global temperature or total ozone over long time scales should be able to duplicate the long-range correlations of temperature and total ozone as shown in DFA analysis. Successful simulation of the DFA curves of observed data would enhance confidence in model predictions of climate and ozone levels.

Acknowledgements. The TOMS data were produced by the Ozone Processing Team at NASA's Goddard Space Flight Center. The ground-based data are credited to Vitaly Fioletov, Experimental Studies Division, Air Quality Research Meteorological Service of Canada. MSU/AMSU data were kindly provided by K. Y. Vinnikov, Department of Atmospheric and Oceanic Science, University of Maryland, USA. Finally, we thank the reviewers and colleagues that commented on our study.

Edited by: M. Dameris

\section{References}

Audit, B., Bacry, E., Muzy, J. F., and Arneodo, A.: Wavelets based estimators of scaling behavior IEEE, Trans. Information Theory 48, 11, 2938-2954, 2002.

Ausloos, M. and Ivanova, K.: Power-law correlations in the southern-oscillation-index fluctuations characterizing El Nino, Phys. Rev. E, 63, 047201, doi:10.1103/PhysRevE.63047201, 2001. 
Blender R. and Fraedrich, K.: Long time memory in global warming simulations, Geophys. Res. Lett. 30, 1769, doi:10.1029/2003GL017666, 2003.

Brandt, J., Christensen, J., Frohn, L. M., and Berkowicz, R.: Air pollution forecasting from regional to urban street scale - implementation and validation for two cities in Denmark, Phys. Chem. Earth, 28(8), 335-344, 2003.

Camp, C. D., Roulston, M. S., and Yung, Y. L.: Temporal and spatial patterns of the interannual variability of total ozone in the tropics, J. Geophys. Res., 108 (D20): art. No. 4643 Oct 25, 2003.

Chandra, S., Varotsos, C., and Flynn, L. E.: The mid-latitude total ozone trends in the northern hemisphere, Geophys. Res. Lett. 23, 555-558, 1996.

Chen, Z., Ivanov, P. C., Hu, K., and Stanley, H. E.: Effect of nonstationarities on detrended fluctuation analysis, Phys. Rev. E, 65, 041107, doi:10.1103/PhysRevE.65.041107, 2002.

Chen, Z., Hu, K., Carpena, P., Bernaola-Galvan, P., Stanley, H. E., and Ivanov, P. C.: Effect of nonlinear filters on detrended fluctuation analysis, Phys. Rev. E, 71, 011104, doi:10.1103/PhysRevE.71.011104, 2005.

Collette, C. and Ausloos M.: Scaling analysis and evolution equation of the North Atlantic oscillation index fluctuations, ArXiv:nlin.CD/0406068 29, 2004.

Crutzen, P. J., Lawrence, M. G., and Pöschl, U.: On the background photochemistry of tropospheric ozone, Tellus A, 51(1), 123-146, 1999.

Dameris, M., Grewe, V., Hein, R., and Schnadt, C.: Assessment of the future development of the ozone layer, Geophys. Res. Lett., 25(19), 3579-3582, 1998.

Dameris, M., Grewe, V., Ponater, M., Deckert, R., Eyring, V., Mager, F., Matthes, S., Schnadt, C., Stenke, A., Steil, B., Bruhl, C., and Giorgetta, M. A.: Long-term changes and variability in a transient simulation with a chemistry-climate model employing realistic forcing, Atmos. Chem. Phys., 5, 2121-2145, 2005, http://www.atmos-chem-phys.net/5/2121/2005/.

Dameris, M., Matthes, S., Deckert, R., Grewe, V., and Ponater, M.: Solar cycle effect delays onset of ozone recovery, Geoph. Res. Lett., 33(3), L03806, doi:10.1029/2005GL024741, 2006.

Ebel, A.: Evaluation and reliability of meso-scale air pollution simulations, Lect. Notes Comput. Sci., 2179, 255-263, 2001.

Fraedrich, K. and Blender, R.: Scaling of atmosphere and ocean temperature correlations in observations and climate models, Phys. Rev. Lett. 90, 108501, doi:10.1103/PhysRevLett.90.108501, 2003.

Galmarini, S., Steyn, D. G., and Ainslie, B.: The scaling law relating world point-precipitation records to duration, Int. J. Climatology, 24(5), 533-546, 2004.

Grytsai, A., Grytsai, Z., Evtushevsky, A., and Milinevsky, G.: Interannual variability of planetary waves in the ozone layer at 65 degrees S, Int. J Remote Sens., 26(16), 3377-3387, 2005.

Hu, K., Ivanov, P. C., Chen, Z., Carpena, P., and Stanley, H. E.: Effect of trends on detrended fluctuation analysis, Phys. Rev. E, 64, 011114, doi:10.1103/PhysRevE.64.011114, 2001.

Hudson, R. D., Frolov, A. D., Andrade, M. F., and Follette, M. B.: The total ozone field separated into meteorological regimes. Part I: Defining the regimes, J. Atmos. Science, 60, 1669-1677, 2003.

Hurst, H. E.: Long term storage capacity of reservoirs. Trans. Am. Soc. Civil Eng., 116, 770-808, 1951.

Jacovides, C. P., Varotsos, C., Kaltsounides, N. A., Petrakis, M., and Lalas, D. P.: Atmospheric turbidity parameters in the highly polluted site of Athens basin, Renewable Energy, 4(5), 465-470, 1994.

Kantelhardt, J. W., Zschiegner, S. A., Koscielny-Bunde, E., Havlin, S., Bunde, A., and Stanley, H. E.: Multifractal detrended fluctuation analysis of nonstationary time series, Physica A, 316(1-4), 87-114, 2002.

Kirk-Davidoff, D. B., Anderson, J. G., Hintsa, E. J., and Keith, D. W.: The effect of climate change on ozone depletion through stratospheric $\mathrm{H}_{2} \mathrm{O}$, Nature, 402, 399, 1999.

Kondratyev, K. Y. and Varotsos, C.: Atmospheric ozone variability: Implications for climate change, human health and ecosystems, Springer, New York, 2000.

Kondratyev, K. Y. and Varotsos, C.: Global tropospheric ozone dynamics - Part I: Tropospheric ozone precursors - Part II: Numerical modelling of tropospheric ozone variability, Environ. Sci. Pollut. Res., 8(1), 57-62 2001a.

Kondratyev, K. Y. and Varotsos, C.: Global tropospheric ozone dynamics - Part II: Numerical modelling of tropospheric ozone variability - Part I: Tropospheric ozone precursors, Environ. Sci. Pollut. Res., 8(2), 113-119 2001b.

Koscielny-Bunde, E. A., Bunde, S. Havlin, H. E. Roman, Y., and Goldreich, Schellnhuber, H. J.: Indication of a universal persistence law governing atmospheric variability. Phys. Rev. Lett., 81(3), 729-732, 1998.

Lawrence, M. G., Crutzen, P. J., Rasch, P. J., Eaton, B. E., and Mahowald, N. M.: A model for studies of tropospheric photochemistry: Description, global distributions, and evaluation, J. Geophys. Res.-Atmos., 104, 26 245-26 277, 1999.

Lovejoy, S.: Area-perimeter relation for rain and cloud areas, Science, 216(4542), 185-187, 1982.

Lovejoy, S. and Schertzer, D.: Direct evidence of multiffractal cascades from planetary scales down to $1 \mathrm{~km}$, Phys. Rev. Lett., 86, 5200, doi:10.1103/PhysRevLett.86.5200, 2001.

Maraun, D., Rust, H. W., and Timmer, J.: Tempting long-memory - on the interpretation of DFA results, Nonlin. Proc. Geoph., 11, 495-503, 2004.

Peng, C. K., Buldyrev, S. V., Havlin, S., Simons, M., Stanley, H. E., and Goldberger, A. L.: Mosaic organization of DNA nucleotides, Phys. Rev. E, 49(2), 1685-1689, 1994.

Richardson, L. F.: Atmospheric diffusion shown on a distanceneighbour graph, Proc. Roy. Soc. A, 110, 709-737, , 1926.

Rust, H.: Interactive comment on "Long-memory processes in global ozone and temperature variations", edited by: Varotsos, C. and Kirk-Davidoff, D., Atmos. Chem. Phys. Discuss. 6, S1182S1185, 2006.

Sander, R.: Modeling atmospheric chemistry: Interactions between gas-phase species and liquid cloud/aerosol particles, Surv. Geophys., 20(1), 1-31, 1999.

Schertzer, D. and Lovejoy, S.: The dimension and intermittency of atmospheric dynamics, in Turbulent Shear Flows, Vol. 4, Springer, New York, 7-33, 1985.

Schulz, A., Rex, M., Harris, N. R. P., Braathen, G. O., Reimer, E., Alfier, R., Kilbane-Dawe, I., Eckermann, S., Allaart, M., Alpers, M., Bojkov, B., Cisneros, J., Claude, H., Cuevas, E., Davies, J., De Backer, H., Dier, H., Dorokhov, V., Fast, H., Godin, S., Johnson, B., Kois, B., Kondo, Y., Kosmidis, E., Kyro, E., Litynska, Z., Mikkelsen, I. S., Molyneux, M. J., Murphy, G., Nagai, T., Nakane, H., O'Connor, F., Parrondo, C., Schmidlin, F. J., Skri- 
vankova, P., Varotsos, C., Vialle, C., Viatte, P., Yushkov, V., Zerefos, C., and von der Gathen, P.: Arctic ozone loss in threshold conditions: Match observations in 1997/1998 and 1998/1999, J. Geophys. Res.-Atmos., 106, 7495-7503, 2001.

Stanley, E.: Scaling, universality, and renormalization: Three pillars of modern critical phenomena, Rev. Mod. Phys., 71(2), S358S366, 1999.

Steinbrecht, W., Hassler, B., Bruhl, C., Dameris, M., Giorgetta, M.A., Grewe, V., Manzini, E., Matthes, S., Schnadt, C., Steil, B., and Winkler, P.: Interannual variation patterns of total ozone and lower stratospheric temperature in observations and model simulations, Atmos. Chem. Phys., 6, 349-374, 2006, http://www.atmos-chem-phys.net/6/349/2006/.

Steinbrecht, W., Hassler, B., Claude, H., Winkler, P., and Stolarski R.S.: Global distribution of total ozone and lower stratospheric temperature variations, Atmos. Chem. Phys., 3, 14211438, 2003, http://www.atmos-chem-phys.net/3/1421/2003/.

Stohl, A., Cooper, O. R., and James, P.: A cautionary note on the use of meteorological analysis fields for quantifying atmospheric mixing, J. Atmos. Sci., 61(12), 1446-1453, 2004.

Syroka, J. and Toumi, R.: Scaling and persistence in observed and modelled surface Temperature, Geophys. Res. Lett., 28(17), 3255-3258, 2001.

Talkner, P. and Weber, R. O.: Power spectrum and detrended fluctuation analysis: Application to daily temperatures, Phys. Rev. E, 62(1), 150-160, 2000.

Toumi, R., Syroka, J., Barnes, C., and Lewis, P.: Robust nonGaussian statistics and long-range correlation of total ozone, Atmos. Sci. Lett., 2(1-4), 94-103, 2001.

Tuck, A. F.: Interactive comment on "Long-memory processes in global ozone and temperature variations", edited by: Varotsos, C. and Kirk-Davidoff, D., Atmos. Chem. Phys. Discuss., 6, S1053S1054, 2006.

Tuck, A. F. and Hovde, S. J.: Fractal behavior of ozone, wind and temperature in the lower stratosphere, Geophys. Res. Lett., 26, 1271-1274, 1999.

Tuck, A. F. Hovde, S. J., and Bui, T. P.: Scale invariance in jet streams:ER-2 data around the lower stratospheric polar night vortex, Q. J. R. Meteorol. Soc., 130, 2423-2444, 2004.

Tuck, A. F., Hovde, S. J., Gao, R. S., and Richard, E. C.: Law of mass action in the Arctic lower stratospheric polar vortex January-March 2000: ClO scaling and the calculation of ozone loss rates in a turbulent fractal medium. J. Geophys. Res.-Atmos., 108(D15), 4451, doi:10.1029/2002JD002832, 2003.

Tuck, A. F., Hovde, S. J., Richard, E. C., Fahey, D. W., Gao, R. S., and Bui, T. P.: A scaling analysis of ER-2 data in the inner Arctic vortex during January-March 2000, J. Geophys. Res.-Atmos., 108(D5), 8306, doi:10.1029/JD000879, 2002.
Varotsos C.: Modern computational techniques for environmental data: Application to the global ozone layer, Proceedings of the ICCS 2005 in Lect. Notes Comput. Sc., 3516, 504-510, 2005.

Varotsos, C. and Kirk-Davidoff, D.: Interactive comment on "Longmemory processes in global ozone and temperature variations" edited by: Varotsos, C. and Kirk-Davidoff, D., Reply to the Comment by: Rust, H., Atmos. Chem. Phys. Discuss., 6, S2602S2605, 2006a.

Varotsos, C. and Kirk-Davidoff, D.: Interactive comment on "Longmemory processes in global ozone and temperature variations", edited by: Varotsos, C. and Kirk-Davidoff, D., Reply to Reviewer \#2, Atmos. Chem. Phys. Discuss., 6, S2613-S2616, 2006b.

Varotsos, C., Kondratyev, K. Y., and Efstathiou, M.: On the seasonal variation of the surface ozone in Athens, Greece, Atmos. Environ., 35(2), 315-320 2001.

Varotsos, P. A., Sarlis, N. V., and Skordas, E. S.: Longrange correlations in the electric signals that precede rupture: Further investigations, Phys. Rev. E, 66, 011902(7), doi:10.1103/PhysRevE.66.011902, 2002.

Varotsos, P. A., Sarlis, N. V., and Skordas, E. S.: Longrange correlations in the electric signals that precede rupture: Further investigations, Phys. Rev. E, 67, 021109, doi:10.1103/PhysRevE.67.021109, 2003a.

Varotsos, P. A., Sarlis, N. V., and Skordas, E. S.: Attempt to distinguish electric signals of a dichotomous nature, Phys. Rev. E, 68, 031106, doi:10.1103/PhysRevE.68.031106, 2003b.

Varotsos, C., Ondov, J., and Efstathiou, M.: Scaling properties of air pollution in Athens, Greece, and Baltimore, Maryland, Atmos. Environ., 39(22) 4041-4047, 2005.

Varotsos, P. A., Sarlis, N. V., Skordas, E. S., Tanaka, H. K., and Lazaridou, M. S.: Entropy of seismic electric signals:Analysis in natural time under time reversal, Phys. Rev. E, 73, 031114, doi:10.1103/PhysRevE.73.031114, 2006.

Vinnikov, K. Y. and Grody, N. C.: Global warming trend of mean tropospheric temperature observed by satellites, Science, 302, 269-272, 2003.

Weber, R. O. and Talkner, P.: Spectra and correlations of climate data from days to decades, J. Geophys. Res., 106, 20 131-20 144, 2001.

World Meteorological Organization: Scientific assessment of ozone depletion 2002, Rep. 47, World Meteorol. Organ., Geneva, Switzerland, 2003. 